\title{
PCFG Parsing for Restricted Classical Chinese Texts
}

\author{
Liang HUANG \\ Department of Computer Science, \\ Shanghai Jiaotong University \\ No. 1954 Huashan Road, Shanghai \\ P.R. China 200030 \\ lhuang@sjtu.edu.cn
}

Huan WANG

Department of Chinese Literature and Linguistics, East China Normal University

No. 3663 North Zhongshan Road, Shanghai, P.R. China 200062

\begin{abstract}
The Probabilistic Context-Free Grammar (PCFG) model is widely used for parsing natural languages, including Modern Chinese. But for Classical Chinese, the computer processing is just commencing. Our previous study on the part-of-speech (POS) tagging of Classical Chinese is a pioneering work in this area. Now in this paper, we move on to the PCFG parsing of Classical Chinese texts. We continue to use the same tagset and corpus as our previous study, and apply the bigram-based forward-backward algorithm to obtain the context-dependent probabilities. Then for the PCFG model, we restrict the rewriting rules to be binary/unary rules, which will simplify our programming. A small-sized rule-set was developed that could account for the grammatical phenomena occurred in the corpus. The restriction of texts lies in the limitation on the amount of proper nouns and difficult characters. In our preliminary experiments, the parser gives a promising accuracy of $82.3 \%$.
\end{abstract}

\section{Introduction}

Classical Chinese is an essentially different language from Modern Chinese, especially in syntax and morphology. While there has been a number of works on Modern Chinese Processing over the past decade (Yao and Lua, 1998a), Classical Chinese is largely neglected, mainly because of its obsolete and difficult grammar patterns. In our previous work (2002), however, we have stated that in terms of computer processing, Classical Chinese is

\author{
Yinan PENG \\ Department of Computer Science, \\ Shanghai Jiaotong University \\ No. 1954 Huashan Road, Shanghai \\ P.R. China 200030 \\ ynpeng@sjtu.edu.cn \\ Zhenyu WU \\ Department of Computer Science, \\ Shanghai Jiaotong University \\ No. 1954 Huashan Road, Shanghai \\ P.R. China 200030 \\ neochinese@sjtu.edu.cn
}

even easier as there is no need of word segmentation, an inevitable obstacle in the processing of Modern Chinese texts. Now in this paper, we move on to the parsing of Classical Chinese by PCFG model. In this section, we will first briefly review related works, then provide the background of Classical Chinese processing, and finally give the outline of the rest of the paper.

A number of parsing methods have been developed in the past few decades. They can be roughly classified into two categories: rule-based approaches and statistical approaches. Typical rule-based approaches as described in James (1995) are driven by grammar rules. Statistical approaches such as Yao and Lua (1998a), Klein and Manning (2001) and Johnson, M. (2001), on the other hand, learn the parameters the distributional regularities from a usually large-sized corpus. In recent years, the statistical approaches have been more successful both in part-of-speech tagging and parsing. In this paper, we apply the PCFG parsing with context-dependent probabilities.

A special difficulty lies in the word segmentation for Modern Chinese processing. Unlike Indo-European languages, Modern Chinese words are written without white spaces indicating the gaps between two adjacent words. And different possible segmentations may cause consistently different meanings. In this sense, Modern Chinese is much more ambiguous than those Indo-European Languages and thus more difficult to process automatically (Huang et al., 2002).

For Classical Chinese processing, such segmentation is largely unnecessary, since most Classical Chinese words are 
single-syllable and single-character formed. To this end, it is easier than Modern Chinese but actually Classical Chinese is even more ambiguous because more than half of the words have two or more possible lexical categories and dynamic shifts of lexical categories are the most common grammatical phenomena in Classical Chinese. Despite of these difficulties, our work (2002) on part-of-speech tagging has shown an encouraging result.

The rest of the paper is organized as follows. In Section 1, a tagset designed specially for Classical Chinese is introduced and the forward-backward algorithm for obtaining the context-dependent probabilities briefly discussed. We will briefly present the traditional two-level PCFG model, the syntactic tagset and CFG rule-set for Classical Chinese in Section 2. Features of the Classical Chinese grammar will also be covered in this section. In Section 3 we will present our experimental results. A summary of the paper is given in the conclusion section.

\section{Tagset and Context-Dependent Probabilities}

Generally speaking, the design of tagset is very crucial to the accuracy and efficiency of tagging and parsing, and this was commonly neglected in the literature where many researchers use those famous corpora and their tagset as the standard test-beds. Still there should be a tradeoff between accuracy and efficiency. In our previous work (2002), a small-sized tagset for Classical Chinese is presented that is shown to be accurate in their POS tagging experiments. We will continue to use their tagset in this paper. We will also use a forward-backward algorithm to obtain the context-dependent probabilities.

\subsection{Tagset}

The tagset was designed with special interest not only to the lexical categories, but also the categories of components, namely subcategories a word may belong. For example, it discriminates adjectives into 4 subcategories like Adjective as attributive, etc. (See table 1). And several grammatical features should be reflected in the tagset. These discriminations and features turn out to be an important contributing factor of the accuracy in our parsing experiments.

Table 1. The tagset for Classical Chinese

\begin{tabular}{|c|c|c|c|}
\hline $\mathrm{n}$ & 名司 & Noun & 楚人有直躬 \\
\hline aล & 形容词作定语 & Adjective as attributive & 营人有直躬 \\
\hline $\mathrm{aw}$ & 形容词作谓语 & $\begin{array}{l}\text { Adjective as verbal } \\
\text { phr ase }\end{array}$ & 被甲者加也 \\
\hline$a b$ & 形容词作表语 & Adjective as predicate & 仲尼以为考 \\
\hline ad & 副词 & Adverb & 慗无用 \\
\hline मे & 不跟宾语的动司 & Verb without object & 知者不感 \\
\hline th & 跟宾语的动词 & Verb with object & 今 学 \\
\hline corij & 便词 & Coryunction & 君子和面不同 \\
\hline $\mathrm{yq}$ & 喵元河 & Exclamation & 被甲者少巴 \\
\hline prep & 带宾语的介词 & Preposition with object & 应之乱则凶 \\
\hline prepb & 省略宾语的介词 & $\begin{array}{l}\begin{array}{l}\text { Preposition with object } \\
\text { omitted }\end{array} \\
\end{array}$ & 仲尼纸之为孝 \\
\hline num & 㖣问 & Number & 虽有 撗帝 \\
\hline gpron & 疑问代词 & Wh-pronoun & 则入事不为也？ \\
\hline ipton & 名词性代词 & Noun-pronoun & 而人主兼礼之。 \\
\hline apron & 形容词性代词 & Adjective-pronoun & 故明主用 勀。 \\
\hline $\mathrm{za}$ & $\begin{array}{l}\text { “之” 作定语后置 } \\
\text { 标志 }\end{array}$ & Special for Old Chinese & 乡人之善者 \\
\hline $\mathrm{zj}$ & “者” 作名词性词尾 & Special for Old Chinese & 乡人之善畨 \\
\hline$z d$ & “之" 作 "的" & Special for Old Chinese & 古之人不嵞欺。 \\
\hline fy & 发语词 & Special for Old Chinese & 太离法者罪。 \\
\hline corigd & 副词性连词 & Adverbial corgunction & 用之維法。 \\
\hline Period & 终止性标点 & & $0 ; ? !$ \\
\hline Comma & 疗顿牲标点 & & , , : \\
\hline
\end{tabular}

\subsection{Tagging Algorithms}

We apply the Hidden Markov Model (HMM) (Viterbi, 1967) and the forward-backward algorithm (James, 1995) to obtain the context-dependent probabilities.

Generally there are 2 types of HMM taggers for parsers, the trigram model and the bigram forward-backward model. Charniak (1996) suggested that the former is better for parsers. 
But the former only result in a deterministic sequence of most probable POS, in other words, it assigns only one POS tag for each word. Although the accuracy of trigram by our previous work (2002) is as high as $97.6 \%$, for a sentence of 10 words long, the possibility of all-correctness is as low as low as $(97.6 \%)^{10}=78.4 \%$, and the single-tag scheme does not allow parsers to re-call the correct tags, as is often done if we apply the forward-backward model. So in this paper we still apply the traditional bigram forward-backward algorithm. We suggest that a combination of trigram and forward-backward model would be the best choice, although no such attempt exists in the literature.

\section{PCFG Model and Classical Chinese Grammar}

In this section we will cover the PCFG model and context-sensitive rules designed for Classical Chinese. Features of the rule-set will be also discussed.

\subsection{PCFG Model and Rule Restriction}

CFG: A context-free grammar (CFG) is a quadruple $\left(V_{N}, V_{T}, S, R\right)$ where $V_{T}$ is a set of terminals (POS tags), $V_{N}$ is a set of non-terminals (syntactic tags), $S \in V_{N}$ is the start non-terminal, and $R$ is the finite set of rules, which are pairs from $V_{N} \times V^{+}$, where $V$ denotes $V_{N} \cup V_{T}$. A rule $\langle A, \alpha>$ is written in the form $A \rightarrow \alpha, A$ is called the left hand side (LHS) and $\alpha$ the right hand side (RHS).

PCFG: A probabilistic context-free grammar (PCFG) is a quintuple $\left(V_{N}, V_{T}, S, R, P\right)$, where $\left(V_{N}, V_{T}, S, R\right)$ is a CFG and $P: R \mapsto(0,1]$ is a probability function such that $\forall N \in V_{N}: \sum_{\alpha: N \rightarrow \alpha \in R} P(N \rightarrow \alpha)=1$

Rule Restriction: We restrict the CFG rules to be binary or unary rules, but NOT as strict as the Chomsky Normal Form (CNF). Each
$R_{i} \in R$ could be in the following two forms only:

1. $R_{i}: N_{j} \rightarrow A B$

2. $R_{i}: N_{j} \rightarrow A$

where $N_{j} \in V_{N}$ and $A, B \in V$

The advantage of binary/unary rules lies in the simplicity of parsing algorithm, and will be discussed in Section 4.

The major difference between our model and $\mathrm{CNF}$ is that for unary rules, we do not require the right-hand-side to be terminals. And this enables us easier representation of the Classical Chinese language.

\subsection{Rule-Set for Classical Chinese}

An important advantage of PCFG is that it needs fewer rules and parameters. According to our corpus, which is representative of Classical Chinese classics, only 100-150 rules would be sufficient. This is mainly because our rule set is linguistically sound. A summary of the set of rules is presented as follows.

Table 2. Our non-terminals (also called syntactic tagset, or constituent set)

\begin{tabular}{|c|c|c|c|}
\hline & Tags & Meaning & Examples \\
\hline 1 & $\mathrm{NP}$ & Noun Phrase & 古之公不余欺 \\
\hline 2 & $\mathrm{VP}$ & Werb Phrase & 古之人不䋇 \\
\hline 3 & $S$ & Sentence & 古之人不条获 \\
\hline 4 & $\mathrm{ADJP}$ & Adjective Phrase & 这人有直躬 \\
\hline 5 & $\mathrm{PP}$ & Prepositional Phrase & 应之 恻则凶 \\
\hline$\overline{6}$ & PADJP & Post-Adjective Phrase & 䏍人之鼓 \\
\hline$?$ & POSTADN & The main part of $\mathrm{PADN}$ & 乡人之锗者 \\
\hline 8 & PREDP & Predicate Phrase & 伸尼以为： \\
\hline
\end{tabular}

A subset of most frequently used rules is shown in the following table. 
Table 3. A simple subset of PCFG Rules for Classical Chinese

\begin{tabular}{|c|c|c|}
\hline S -> & NP VP & ; simple S/V \\
\hline S -> & VP & ; S omitted \\
\hline S -> & VP NP & ; S/V inversion \\
\hline S -> & ad S & \\
\hline VP -> & vi & \\
\hline VP -> & vt NP & ; simple V/O \\
\hline VP -> & $\mathrm{NP} v \mathrm{vt}$ & ; V/O inversion \\
\hline VP $\rightarrow$ & ad VP & \\
\hline VP -> & PP VP & ; prepositioned PP \\
\hline VP -> & VP PP & ; postpositioned PP \\
\hline VP $->$ & NP & ; NP as VP \\
\hline VP $\rightarrow$ & VP yq & \\
\hline$N P$-> & $\mathrm{n}$ & \\
\hline
\end{tabular}

\begin{tabular}{|c|c|c|c|}
\hline 14. & NP $\rightarrow>$ & npron & \\
\hline 15. & NP -> & ADJP NP & \\
\hline 16. & $N P$-> & POSTADJH & \\
\hline 17. & NP -> & VP & ; V/O as NP \\
\hline 18. & $\mathrm{NP}->$ & fy NP & \\
\hline 19. & ADJP -> & aa & \\
\hline 20. & ADJP -> & apron & \\
\hline 21. & ADJP -> & NP zd & \\
\hline 22. & PP -> & prep NP & ; P+NP \\
\hline 23. & PP -> & NP prep & ; inversion \\
\hline 24. & PP -> & prepb & ; object omitted \\
\hline 25. & PP -> & NP & ; prep. omitted \\
\hline 26. & POSTADJP-> & VP zj & \\
\hline
\end{tabular}

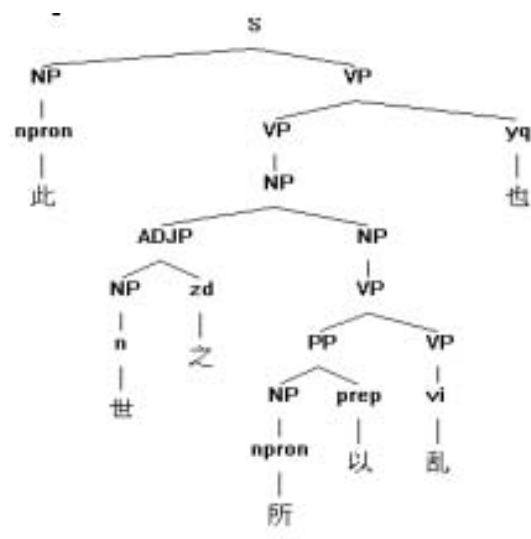

(a)

Examples of parse trees are shown in the following figure.

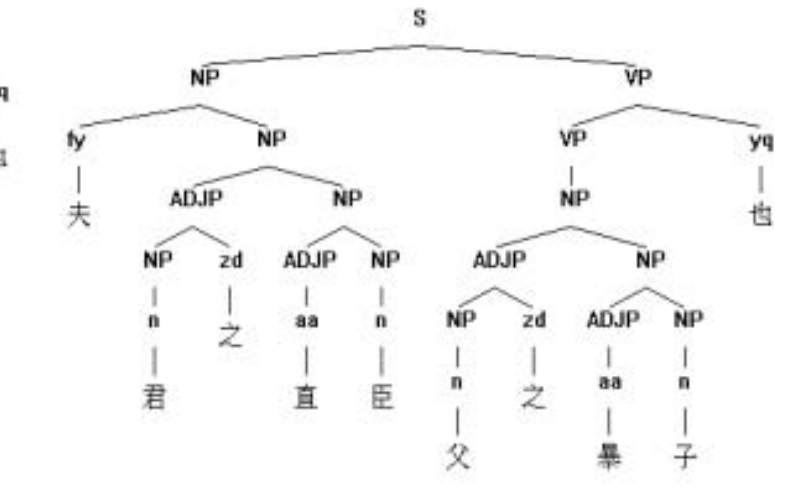

(b)

Fig. 1. the parse trees of 2 sentences

(a) 此世之所以乱也。 (b) 夫君之直臣父之暴子也。

\subsection{Features of Classical Chinese Grammar Rules}

As an aside, it is worthwhile to point out here some peculiarities of the Classical Chinese grammar used in our work. Readers not interested in grammar modeling may simply skip this subsection. As mentioned before, the grammar of Classical Chinese is entirely different from that of English, so a few special features must be studied. Although these features bring many difficulties to the parser, we have developed successful programming techniques to solve them.
From the rule-set, the reader might find that two special grammatical structures is very common in Classical Chinese:

1. Inversion: subject/verb inversion (rule 3), preposition/object inversion (rule 23).

2. Omission: Subject omitted (rule 2), preposition's object omitted (rule 24), preposition omitted (rule 25).

Maybe the strangest feature is the structure of PP. English PP is always P+NP. But here in Classical Chinese, by inversion and omission, the PP may have up to 4 forms, as shown in rule 22-25. 
Table 4. The 4 rules from PP. The object of the preposition is in brackets, and [] indicate an omission.

\begin{tabular}{|c|c|c|c|}
\hline & Rule & Explanation & Example \\
\hline 22 & PP $>$ prep NP & The normal $\mathrm{P}+\mathrm{NP}$ & 儒 以(文)乱法。 \\
\hline 23 & PP $>$ NP prep & $\begin{array}{l}\text { Inverted: NP+P } \\
\text { (介词宾语前置) }\end{array}$ & 此(央)咶代。 \\
\hline 24 & PP $>$ prepb & $\begin{array}{l}\text { The object of the } \\
\text { preposition is } \\
\text { omitted }\end{array}$ & 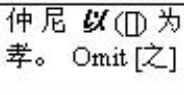 \\
\hline 25 & $\mathrm{PP} \gg \mathrm{NP}$ & $\begin{array}{l}\text { The preposition } \\
\text { itself is omitted }\end{array}$ & $\begin{array}{l}\text { 谒之口(吏)。 } \\
\text { omit [于] }\end{array}$ \\
\hline
\end{tabular}

Another feature that must be pointed out here is the cycle. In our rule-set, there are 2 rules (rule 11 and rule 17) forming a cycle:

$$
\mathrm{NP} \underset{17}{\stackrel{11}{\longrightarrow} \mathrm{VP}}
$$

Fig. 2. A cycle in the rule-set. Rule 11: NP-> VP, Rule 17: VP $\rightarrow$ NP.

It will ease our parsing because Classical Chinese is lexically and syntactically very ambiguous. An NP can act as a VP (a main verb), while a VP can act as a NP (subject or object). These two features are exemplified in figure 3 . There are actually more cycles in the rule-set. Helpful as they are, the cycles bring great difficulty to the memory-based top-down parser. In practice, we develop a closure-based method to solve this problem, as shown in the following pseudo-code:

better_results_found=true;

while (better_results_found)

better_results_found=false; memory_based_top_down_parse(); \} // if better results found, the variable will be set true

Another point is the use of preferences for ambiguity resolution. While the ambiguities in our rule-set greatly ease our modeling Classical Chinese grammar, it causes the parser to make a lot of ridiculous errors. So we here apply some predefined preferences such as 'an fy must be at the first of an NP' and 'a yq must be at the end of a VP'. This consideration results in a significant increase in the parsing accuracies.

\section{Evaluations}

In our preliminary experiments, we constructed a treebank of 1000 manually parsed sentences (quite large for Classical Chinese treebank), in which 100 sentences are selected as the test set using the cross-validation scheme, while the others as the learning set. The majority of these sentences are extracted from classics of pre-Tsin Classical Chinese such as Hanfeizi and Xunzi because in these texts there are fewer proper nouns and difficult words. That is the restriction we put on the selection of Classical Chinese texts. It must be pointed out here that compared from other languages, Classical Chinese sentences are so short that the average length is only about 4-6 words long.

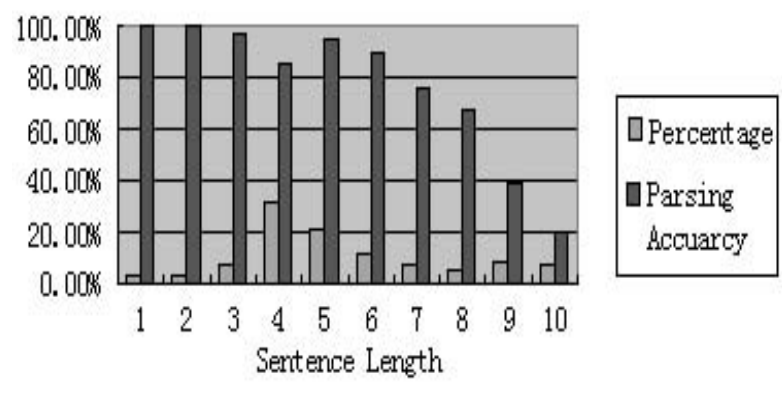

Fig. 3. Sentence Distributions and Parsing Accuracies

Figure 3 shows the distribution of sentences and parsing accuracies for different sentence lengths. For distribution, we can see that those 4-word, 5-word, and 6-word sentences constitute for the majority of the corpus, while those 1-word and 2-word sentences are very few. For accuracy, the parser is more effective for shorter sentences than for longer sentences. And for 1-word and 2-word sentences, there is no error report from the parse results. 


\section{Conclusion}

Computer processing of Classical Chinese has just been commencing. While Classical Chinese is generally considered too difficult to process, our previous work on part-of-speech tagging has been largely successful because there is almost no need to segment Classical Chinese words. And we continue to use the tagset and corpus into this work. We first apply the forward-backward algorithm to obtain the context-dependent probabilities. The PCFG model is then presented where we restrict the rules into binary/unary rules, which greatly simplifies our parsing programming. According to the model, we developed a CFG rule-set of Classical Chinese. Some special features of the set are also studied. Classical Chinese processing is generally considered too difficult and thus neglected, while our works have shown that by good modelling and proper techniques, we can still get encouraging results. Although Classical Chinese is currently a dead language, our work still has applications in those areas as Classical-Modern Chinese Translation.

For future work of this paper, we expect to incorporate trigram model into the forward-backward algorithm, which will increase the tagging accuracy. And most important of all, it is obvious that the state-of-the-art PCFG model is still two-leveled, we expect to devise a three-level model, just like trigram versus bigram.

\section{Acknowledgements}

Our special thanks go to Prof. Lu Ruzhan of Shanghai Jiaotong University for his sincere guidance.

\section{References}

Allen, J. (1995) Natural Language Understanding, The Benjamin/Cummings Publishing Company, Inc.

Viterbi, A. (1967) Error bounds for convolution codes and an asymptotically optimal decoding algorithm. IEEE Trans. on Information Theory 13:260-269.

Yao Y., Lua K. (1998a) A Probabilistic Context-Free Grammar Parser for Chinese, Computer Processing of Oriental Languages, Vol. 11, No. 4, pp. 393-407

Huang L., Peng Y., Wang H. (2002) Statistical Part-of-Speech Tagging for Classical Chinese, Proceedings of the $5^{\text {th }}$ International Conference on Text, Speech, and Dialog (TSD), Brno, in press

Klein, D., and Manning C. (2001) Natural Language Grammar Induction using a Constituent-Context Model, Proceedings of Neural Information Processing Systems, Vancouver.

Yao Y., Lua K. (1998b) Mutual Information and Trigram Based Merging for Grammar Rule Induction and Sentence Parsing, Computer Processing of Oriental Languages, Vol. 11, No. 4, pp. 393-407

Johnson, M. (2001) Joint and conditional estimation of tagging and parsing models, Proceedings of International computational linguistics conference, Toulouse

Charniak, E. (1996) Taggers for Parsers, Artificial Intelligence, Vol. 85, No. 1-2, pp. 45-47. 